\title{
Analysis of transport properties of iron pnictides: spin-fluctuation scenario
}

\author{
P. Prelovšek ${ }^{1,2}$, I. Sega ${ }^{1}$ and T. Tohyama ${ }^{3}$ \\ ${ }^{1}$ J. Stefan Institute, SI-1000 Ljubljana, Slovenia \\ ${ }^{2}$ Faculty of Mathematics and Physics, University of Ljubljana, SI-1000 Ljubljana, Slovenia and \\ ${ }^{3}$ Yukawa Institute for Theoretical Physics, Kyoto University, Kyoto 606-8502, Japan
}

(Dated: August 27, 2018)

\begin{abstract}
We present a phenomenological theory of quasiparticle scattering and transport relaxation in the normal state of iron pnictides based on the simplified two-band model coupled via spin fluctuations. In analogy with anomalous properties of cuprates it is shown that a large and anomalous normal-state resistivity and thermopower can be interpreted as the consequence of strong coupling to spin fluctuations. The generalization to the superconducting phase is also discussed.
\end{abstract}

PACS numbers: 71.27.+a, 75.20.-g, 74.72.-h

\section{INTRODUCTION}

Recently discovered superconducting (SC) iron pnictides [1, 2] are at present in the focus of experimental and theoretical investigations in the solid state community. Besides high $\mathrm{SC} T_{c}$ the main motivation is the fact that a wide class of materials opens yet another view on the interplay of magnetism and SC in metals. At present it seems that there is firm evidence that the physics of novel materials is not in the class of strongly correlated systems close to the Mott-Hubbard insulator as is the case in SC cuprates [3], investigated intensively in the last two decades. On the other hand there are challenging similarities, in particular in the anomalous transport properties [4, 5, 6], in the presumable unconventional type of SC [7, 8], as well as in the importance of spin fluctuations apparently evidenced by the NMR relaxation [9] and the onset of spin-density-wave (SDW) order [10].

In this paper we put the emphasis on the transport properties of iron pnictides (IP) and their understanding. Published experimental results on the d.c. electrical resistivity $\rho(T)$ generally reveal high values at $T>T_{c}$, comparable in magnitude to underdoped cuprates [11]. At the same time, at elevated $T \sim 300 \mathrm{~K}$ the $T$-dependence is linear with large slope $d \rho / d T$, again similar to the well known anomalous variation in cuprates. Since the systematics of different compounds is still not fully understood we concentrate here on the family emerging from the reference (undoped) compound LnFeAsO (LFAO) where besides the original $\mathrm{Ln}=\mathrm{La}$ a variety of other lantanides $\mathrm{Ln}=\mathrm{Ce}$ - Dy has been investigated so far. The electron doping has been studied either by doping with $\mathrm{F}$, i.e., in $\mathrm{LnFeAsO}_{1-x} \mathrm{~F}_{x}$ [4, 5, 6] or via oxygen deficiency $\mathrm{LnFeAsO}_{1-y}[12]$. The evidence so far is that different Ln do show similar results, while the resistivity $\rho(T)$ reveals quite systematic and universal change with doping $x$ [6] or $y$ [12]. E.g., the resistivity [6, 12] changes from that of the SDW semimetal at $x, y<0.05$ with large $\rho\left(T>T_{S D W}\right)$, a property shared in particular by underdoped cuprates, over the intermediate (optimally doped) regime $x, y \sim 0.1$ with a quite pronounced linear law $\rho \propto T$, into the overdoped regime with more Fermi-liquid (FL) $T^{2}$ behavior for $y>0.2$.

Also the thermopower $S(T)$ is far from the FL behavior
$S \propto T$ [4, 5], reaching at $T>T_{c}$ values characteristic for nondegenerate electrons, i.e., $|S| \sim s_{0}=k_{B} / e_{0}=86 \mu \mathrm{V} / \mathrm{K}$ again being a remarkable property of underdoped cuprates [13]. Similar message is emerging from strongly $T$-dependent Hall constant $R_{H}(T)$ [14]. So far, there are very few data on dynamical transport properties, nevertheless optical conductivity in the same system [15] seems to support non-Drudelike relaxation with large and $\omega$-dependent transport relaxation rate $\tau^{-1} \sim \omega$.

A detailed analysis of transport data on IP seems to be still premature due to mostly polycrystalline samples studied so far as well as due to the lack of doping systematics in the electron subsystem. Nevertheless in the following we argue that the similarity to cuprates arises from the strong coupling to spin fluctuations and the non-FL-like behavior following the marginal FL (MFL) scenario [16]. More specific origin of spin fluctuations and the spin-fermion coupling could be, however, quite different, e.g., due to the importance of the Hund's rule coupling $J_{H}$, also shown to lead to large $\rho(T)$ [17. Also, emerging novel results on single-crystal transport obtained mostly by the electron doping the reference compound $\mathrm{BaFe}_{2} \mathrm{As}_{2}$ [18, 19] require a quantitative reconsideration, not followed in this work in detail, due to observed higher mobilities. Nevertheless, several main characteristics as the linear $\rho(T)$ law, large and anomalous thermopower $S(T), T$-dependent Hall constant $R_{H}(T)$ again confirm our basic assumptions given below.

\section{A SIMPLIFIED MODEL}

A microscopic model for relevant electrons in IP appears to be quite complicated and still debated at present [8]. While it has been argued that a (minimal) two-band model already contains the essential low energy physics in these materials $[20,21,22]$, the inadequacy of certain approximations to the effective low-energy band structure has been recently criticized [23]. Nevertheless, for the purpose of our qualitative analysis we employ the model with two bands, one electronlike and another hole-like, coupled, however, through spin fluctuations introduced phenomenologically [24] and treated 
within the lowest-order pertubation theory. It is evident that such a simplified model is not enough to describe calculated band structure [25] as well as the one observed via angle resolved photoemission [26] or via the de Haas-van Alphen effect [27], revealing (at least) four pockets at the Fermi surface in the actual Brillouin zone. However, in the simplest approach to spin-fluctuation mechanism it is essential that SDWtype spin fluctuation inter-couple electron and hole bands, while the coupling among electron (hole) bands themselves should be less important.

In contrast to IP, a vast experimental evidence in the last two decades shows that cuprates can be well modeled with a single-band model [3], e.g., the simple 2D Hubbard model or the $t-J$ model [28] being, however, in the regime of strong correlations which can be only approximately described with a coupled spin-fermion model [24]. Starting from the latter level assuming the MFL behavior of spin fluctuations and a strong coupling to electrons yield in cuprates anomalous quasiparticle (QP) damping [29] and transport relaxation [30] as well as an unconventional SC [29].

We use a simplified model for IP describing the 2D system with one electron (e) band and the other hole (h) band crossing the Fermi surface [8, 20, 21]. I.e., in the (folded) Brillouin zone [8, 22] the h-like and e-like pockets are at $k \sim 0$, and $\mathbf{k} \sim \mathbf{Q}=(\pi, \pi)$, respectively. Within this effective model bands are coupled only via spin fluctuations, leading to

$$
\begin{aligned}
H_{e f}= & -\sum_{\mathbf{k}, s}\left(\zeta_{\mathbf{k}}^{e} c_{\mathbf{k} s}^{\dagger} c_{\mathbf{k} s}+\zeta_{\mathbf{k}}^{h} d_{\mathbf{k} s}^{\dagger} d_{\mathbf{k} s}\right)+ \\
& \frac{1}{\sqrt{N}} \sum_{\mathbf{k q}, s s^{\prime}} m_{\mathbf{k q}} \mathbf{S}_{\mathbf{q}} \cdot \sigma_{s s \prime}\left(c_{\mathbf{k}-\mathbf{q}, s}^{\dagger} d_{\mathbf{k} s^{\prime}}+d_{\mathbf{k}-\mathbf{q}, s}^{\dagger} c_{\mathbf{k} s^{\prime}}\right),(1)
\end{aligned}
$$

and $c_{\mathbf{k}}, d_{\mathbf{k}},\left(\zeta^{e}, \zeta^{h}\right)$ refer to electrons in e-like and h-like bands, respectively. We consider the corresponding Green's functions for e- and h-electrons

$$
G_{\mathbf{k}}^{\sigma}(\omega)=\left[\omega^{+}-\epsilon_{\mathbf{k}}^{\sigma}-\Sigma_{\mathbf{k}}^{\sigma}(\omega)\right]^{-1},
$$

where $\epsilon_{\mathbf{k}}^{\sigma}=\zeta_{\mathbf{k}}^{\sigma}-\mu$, and $\sigma=e, h(\bar{\sigma}=h, e)$.

\section{QUASIPARTICLE DAMPING}

Within the lowest order perturbation theory [29, 36] the self energies can be expressed as

$$
\begin{aligned}
\Sigma_{\mathbf{k}}^{\sigma}(\omega) & =3 \sum_{\mathbf{q}} m_{\mathbf{k q}}^{2} \iint \frac{d \omega_{1} d \omega_{2}}{\pi} g_{12} \frac{A_{\mathbf{k}-\mathbf{q}}^{\bar{\sigma}}\left(\omega_{1}\right) \chi_{\mathbf{q}}^{\prime \prime}\left(\omega_{2}\right)}{\omega-\omega_{1}-\omega_{2}} \\
g_{12} & \equiv g\left(\omega_{1}, \omega_{2}\right)=\frac{1}{2}\left[\operatorname{th} \frac{\beta \omega_{1}}{2}+\operatorname{cth} \frac{\beta \omega_{2}}{2}\right]
\end{aligned}
$$

where $\chi_{\mathbf{q}}(\omega)$ is the dynamical spin susceptibility.

To proceed we make several simplifications, which are expected to be a reasonable starting point for a qualitative analysis of transport quantities in IP. Spin response close to the antiferromagnetic (SDW) instability [10] centered at $\mathbf{q} \sim \mathbf{Q}=(\pi, \pi)$ we assume broad enough relative to $\mathrm{h} / \mathrm{e}$ pockets to replace $\chi_{\mathbf{q}}(\omega) \sim \chi_{\mathbf{Q}}(\omega)=\tilde{\chi}(\omega)$. In this case $\Sigma_{\mathbf{k}}^{e} \sim \Sigma_{\mathbf{Q}}^{e}=\Sigma^{e}$ and $\Sigma_{\mathbf{k}}^{h} \sim \Sigma_{\mathbf{0}}^{h}=\Sigma^{h}$, and the QP damping is $\Gamma^{\sigma}(\omega)=-\operatorname{Im} \Sigma^{\sigma}(\omega)$.

It should be however pointed out that the momentum dependence of the self energy could be important or even crucial. The latter would be the case if, e.g., the (accurate) nesting would play a role. Since anomalous behavior of IP seems to be quite robust, e.g., linear resistivity over a broad $T$ and doping range, large thermopower $S(T)$ as well as high $T_{c}$, we believe that this is not the case so that momentum dependence along the Fermi pockets is not crucial also requiring $\chi_{\mathbf{q}}(\omega)$ response not too narrow in $\mathbf{q}$.

With the above simplifications we get for the QP damping

$$
\Gamma^{\sigma}(\omega)=\frac{3}{2} \lambda \int d \omega^{\prime} g\left(\omega-\omega^{\prime}, \omega^{\prime}\right) \mathcal{N}^{\bar{\sigma}}\left(\omega-\omega^{\prime}\right) \tilde{\chi}^{\prime \prime}\left(\omega^{\prime}\right),
$$

where $m_{\mathbf{Q}, \mathbf{Q}}=m_{\mathbf{0}, \mathbf{Q}}=\bar{m}, \lambda=\bar{m}^{2}$, and $\mathcal{N}^{\sigma}(\omega)$ are the $\sigma$-band density of states (DOS). We also get

$$
A_{\mathbf{k}}(\omega)=-\operatorname{Im}\left[\Omega(\omega)-\epsilon_{\mathbf{k}}+i \Gamma(\omega)\right]^{-1},
$$

where $\Omega(\omega)=\omega-\operatorname{Re} \Sigma(\omega)=\omega Z^{-1}(\omega)$ defines the QP weight $Z(\omega)$.

In analogy with cuprates, large and non-FL-like linear resistivity $\rho(T) \propto T$ and a presumable transport relaxation rate $\tau^{-1} \propto \omega$ in particular, require also a MFL behavior [16] of the spin fluctuation input $\chi(\omega)$. In our analysis MFL-type spin fluctuations are a phenomenological assumption which still has to be confirmed by specific experiments as, e.g., the inelastic neutron scattering. It is also fair to admit that a theoretical understanding of such spin fluctuations in IP is not yet available, whereby even for cuprates there is not yet an agreement on the explanation for the MFL physics. Still, as for cuprates there are no accepted alternative scenarios for the non-FL behavior of QP damping and transport quantities.

Hence, we employ the relation [28]

$$
\tilde{\chi}^{\prime \prime}(\omega)=\pi \bar{C}(\omega) \operatorname{th}(\beta \omega / 2),
$$

where $\bar{C}(\omega)=C(\omega)+C(-\omega)$ and $C(\omega)$ represents the dynamical spin correlation function. In analogy with the MFL scenario [16] well established for cuprates we use a $T$ independent ansatz $C(\omega) \sim C_{0}, \omega<\omega_{0} \gg T[28,30]$. It should be noted that such an ansatz by construction satisfies the $T$-independent sum rule [31] given by

$$
\frac{1}{\pi} \int_{0}^{\infty} \operatorname{cth} \frac{\beta \omega}{2} \tilde{\chi}^{\prime \prime}(\omega) d \omega=\int_{0}^{\infty} \bar{C}(\omega) d \omega=\left\langle S_{i}^{z} S_{i}^{z}\right\rangle,
$$

In underdoped cuprates the MFL behavior emerging from the above assumptions is qualitatively rather well established both experimentally [3, 32] and from model calculations [28], although other forms close to Eq.(6) have also been proposed [32]. The origin of a non-FL behavior seems to emerge from the fact that due to the localized character of spins the low$\omega$ spin-fluctuations exhaust the sum rule [31]. This is much 
less evident for IP but still appears to be a prerequisite for the MFL-like behavior of transport quantities as described furtheron.

Assuming in the relevant regime $\mathcal{N}(\omega) \sim \mathcal{N}$ (being to lowest order unchanged even if the QP weight $Z<1$ ) we get

$$
\Gamma^{\sigma}(\omega)=\gamma^{\sigma} \omega \operatorname{coth}(\beta \omega / 2), \gamma^{\sigma}=(3 \pi / 2) \lambda C_{0} \mathcal{N}^{\bar{\sigma}},
$$

i.e., approximately

$$
\Gamma^{\sigma}(\omega) \cong \gamma^{\sigma} \max (|\omega|, 2 T) .
$$

However, for further interpretation, in particular of the Seebeck coefficient $S(T)$, it seems essential that the DOS be nonsymmetric around $\omega \sim 0$. E.g., a possible assumption is $\mathcal{N}(\omega \gtrless 0) \sim \mathcal{N}_{ \pm}$, where the DOS can differ for $\omega \gtrless 0$ and consequently $\gamma^{\sigma} \rightarrow \gamma_{ \pm}^{\sigma}$. While the asymmetry of the relaxation rate seems to be the only viable explanation for the large $S(T)$, assumptions are expected to emerge from a more detailed analysis of $\mathcal{N}(\omega \sim 0)$ in a doped semimetal and corresponding scattering rates for $\omega \pm 0$. E.g., due to very shallow hole bands at $\omega>0$ for electron scattering in e-doped IP [26, 33] as considered below one is effectively dealing with different $\mathcal{N}_{ \pm}$.

\section{TRANSPORT QUANTITIES}

Turning to transport properties, we first consider optical conductivity $\sigma(\omega)$ which we assume isotropic within the FeAs, i.e., easy plane. Within the linear response (neglecting vertex corrections) $\sigma(\omega)$ can be expressed as [34]

$$
\begin{aligned}
& \sigma(\omega)=\frac{2 \pi e_{0}^{2}}{N \omega} \sum_{\mathbf{k}, \sigma}\left(v_{\mathbf{k} \sigma}^{x}\right)^{2} \times \\
& \times \int d \omega^{\prime}\left[f\left(\omega^{\prime}-\omega\right)-f\left(\omega^{\prime}\right)\right] A_{\mathbf{k}}^{\sigma}\left(\omega^{\prime}\right) A_{\mathbf{k}}^{\sigma}\left(\omega^{\prime}-\omega\right),
\end{aligned}
$$

where $v_{\mathbf{k} \sigma}^{x}$ are the corresponding band velocities. In the following we consider e-doped IP, therefore for simplicity we take as dominant the e-pocket contribution. Everywhere refering to the e-band and defining the function

$$
\Phi(\epsilon)=\frac{2 e_{0}^{2}}{N} \sum_{\mathbf{k}}\left(v_{\mathbf{k}}^{x}\right)^{2} \delta\left(\epsilon-\epsilon_{\mathbf{k}}\right)
$$

we get for slowly varying $\Phi(\epsilon) \sim \Phi_{0}$

$$
\sigma(\omega)=\Phi_{0} \frac{1-e^{-\beta \omega}}{\omega} \int d \omega^{\prime} \frac{f\left(-\omega^{\prime}\right) f\left(\omega^{\prime}-\omega\right) F_{12}}{\bar{\Omega}_{12}^{2}+F_{12}^{2}},
$$

where $F_{12}=\Gamma\left(\omega^{\prime}\right)+\Gamma\left(\omega^{\prime}-\omega\right)$ and $\bar{\Omega}_{12}=\Omega\left(\omega^{\prime}\right)-\Omega\left(\omega^{\prime}-\right.$ $\omega) \sim Z^{-1} \omega$.

For $\sigma(\omega \gg T)$ one gets from Eq.(12) the extended Drude form

$$
\sigma(\omega \gg T)=\tilde{\Phi} \frac{\Gamma_{t r}(\omega)}{\omega^{2}+\Gamma_{t r}^{2}(\omega)}
$$

and $\tilde{\Phi}=Z \Phi_{0} \sim n_{e} e_{0}^{2} / m_{e}^{*}$, with $m_{e}^{*}$ the QP mass in the e-pocket. Assuming further the MFL form for $\Gamma(\omega)$, Eq.(8), the effective transport relaxation rate $\Gamma_{t r}(\omega)=\tilde{\gamma}_{a} \omega$ where $\tilde{\gamma}_{a}=Z\left(\gamma_{+}+\gamma_{-}\right) / 2$.

For the d.c. conductivity Eq. (12) reduces to [34, 35]

$$
\sigma(0)=\tilde{\Phi} \int d \omega\left(-\frac{\partial f}{\partial \omega}\right) \frac{1}{\Gamma_{t r}(\omega)},
$$

which, on assuming constant $\tilde{\Phi}$ and MFL-type $\Gamma$, Eq.(8), immediately yields linear-in- $T$ resistivity

$$
\rho=\frac{T}{A_{0}}=\frac{T}{\tilde{\Phi} \tilde{A}_{0}} .
$$

Within the same local approximation for $\Sigma_{\mathbf{k}}(\omega)=\Sigma(\omega)$ also the Seebeck coefficient $S$ can be expressed as [35]

$$
S=-w s_{0}, \quad w=\tilde{A}_{1} / \tilde{A}_{0},
$$

where

$$
\tilde{A}_{n}=T \int d \omega\left(-\frac{\partial f}{\partial \omega}\right) \frac{(\beta \omega)^{n}}{2 Z \Gamma(\omega)} .
$$

Under the MFL assumption for $\Gamma(\omega)$, all $\tilde{A}_{n}$ are $T$ independent and in contrast to the FL behavior $S \propto T$ one gets a $T$-independent $S \sim$ const.

In the symmetric case $\gamma_{-}=\gamma_{+}=\gamma$ and by Eqs.(8), (17)

$$
\tilde{A}_{0}=0.21 / \tilde{\gamma},
$$

while $A_{1}=0$ identically and therefore $S(T)=0$. It is thus evident that a pronounced asymmetry in $\Gamma(\omega)$ is needed to explain large $S(T)$ in IP, as discussed later. One situation possibly relevant for e-doped IP is that $\gamma_{-} \gg \gamma_{+}$. This can happen if upon electron doping the h-pocket states diminish substantially at $\omega>0$ as a source of scattering, leading to the reduction of $\gamma_{+}$. In such a limiting case we get

$$
\tilde{A}_{0}=0.10 / \tilde{\gamma}_{+}, \quad w \sim 1.2 .
$$

\section{ANALYSIS OF EXPERIMENTAL RESULTS}

So far, most information for the normal(N)-state transport in IP is available for the d.c. resistivity $\rho(T)$. We analyse here only the data for electron doped LFAO compounds. The whole range of $x$ for F-doped LFAO has been measured recently [6], while $S(T)$ as well as Hall coefficient $R_{H}(T)$ have been measured also for $x=0.11$ [4, 5] and $x=0.05$ [14]. It should be reminded, however, that all data so far are for polycrystals while the theory is done for the transport oriented along the easy plane, so that measured $\rho(T)$ should be at least scaled by some factor $\xi<1$ to get the relevant planar resistivity considered here.

First, it is evident that inverse mobilities are larger in IP as compared to cuprates. Thus in $\operatorname{LaSr}_{x} \mathrm{CuO}_{1-x}$ at $T=300 \mathrm{~K}$ and for doping $x=0.03-0.1$ [11],

$$
\mu^{-1}=n_{h} e_{0} \rho_{a b}=(0.3-0.15) \mathrm{Vs} / \mathrm{cm}^{2} .
$$


Analogous results for IP compounds depend on the density $\bar{x}$ of carriers/formula unit, where $n_{e}=\bar{x} / V_{0}$ and $V_{0}$ is the volume of a formula unit. Even undoped $x=0$ IP have finite but small $n_{e}^{0}$, i.e., $x_{0}>0$ and it is plausible that $\bar{x}=x_{0}+x>$ $x$. From data at $T=300 \mathrm{~K}[6]$ we get

$$
\mu^{-1}=n_{e} e_{0} \rho(T) \sim(5.6-8.1) \bar{x} \mathrm{Vs} / \mathrm{cm}^{2},
$$

for $x=0.05-0.2$, respectively. Similar results are obtained for $x=0.11$ [4, 5] $\mu^{-1}=5.8 \bar{x} \mathrm{Vs} / \mathrm{cm}^{2}$, and for $x=0.05$ [14] $\mu^{-1}=8.0 \bar{x} \mathrm{Vs} / \mathrm{cm}^{2}$. It is evident that such $\mu^{-1}$ are even higher than in low-doped cuprates [11].

Existing data for $\rho(T)$ in LFAO show a large slope at higher $T \gg T_{c}$, also with a larger onset $\rho\left(T \sim T_{c}\right)$ at $x=0.05$, while for $x>0.1 \rho\left(T<T^{*} \sim 100 K\right)$ becomes nonlinear and more FL-like. To estimate $\tilde{A}_{0}$ and consequently $\tilde{\gamma}$ we use the slope at $T<300 \mathrm{~K}$, i.e., $\tilde{A}_{0}^{-1}=\tilde{\Phi} d \rho(T) / d T$ and we get for a wide range of $x$ [6]

$$
\tilde{A}_{0} \sim \alpha \nu / \bar{x}, \quad \alpha \sim 3.8 \cdot 10^{-3}, \quad \tilde{\gamma} \sim 56 \bar{x} / \nu,
$$

where $\nu=m / m^{*}$. Similar values are obtained analysing other data for F-doped LFAO: for $x=0.11$ [5] and for $x=0.05$ we get $\alpha \sim 4.0 \cdot 10^{-3}$ [14]. Not much different is the development of $\rho(T)$ for the oxygen deficient LFAO [12] where $y<0.03$ compounds reveal a non-SC state with a SDW transition $T_{S D W}>0$ while for $y>0.1$ again $\rho(T)$ is nicely linear in $T$ with $\alpha \sim 0.006$. Assuming, e.g., values from band structure calculations $\nu \sim 2$ and $\bar{x} \sim 0.1$ we arrive at very large $\tilde{\gamma} \sim 2.8$. Such value $\tilde{\gamma}$ is most likely an overestimate due to too large $\rho$ (all cited measurements are for polycrystals) and possibly due to relatively small QP mass enhancement $\nu \sim 2$.

So far, there are only few data for optical conductivity $\sigma(\omega)$. In the LFAO with As replaced by P $\sigma(\omega)$ was measured and analysed [15] using the extended Drude fit yielding for $\omega<1000 \mathrm{~cm}^{-1}$ anomalous $\tau^{-1}=\Gamma_{t r} \sim \omega$, i.e., $\tilde{\gamma} \sim 1$ qualitatively consistent with the above estimates.

Experimental results for $S(T)$ for LFAO yield typically elike $S<0$ with strong $T$-dependence with the maximum values $S \sim-s_{0}$ at $T \sim 100 \mathrm{~K}$. Assuming $\Delta \tilde{\gamma}=\tilde{\gamma}_{-}-\tilde{\gamma}_{+}>\tilde{\gamma}_{+}$ we get from Eq. (17)

$$
w \sim \Delta \tilde{\gamma} /\left(12 \tilde{\gamma}_{a}\right)
$$

whereas in the extreme asymmetric limit, Eq. 19, we recover $w=1.2$.

\section{SUPERCONDUCTIVITY}

Let us finally comment on the relation of the above analysis to the treatment of the SC phase within the assumption of the spin-fluctuation induced pairing. We follow here closely the treatment of SC within the effective spin-fermion model [29, 36] as derived from the microscopic strong-correlation model (planar $t-J$ model) relevant for cuprates. Since our paper is not focused on the question of SC in IP, the aim is to connect parameters entering transport quantities to those determining the SC gaps and consequently $T_{c}$.

For the discussion of SC equations can be generalized with Green's functions and selfenergies being $2 \times 2$ matrices [36] whereby we (again) neglect e/h interband terms

$$
\mathbf{G}_{\mathbf{k}}^{\sigma}(\omega)=\left[\omega \tau_{0}-\epsilon_{\mathbf{k}}^{\sigma} \tau_{1}-\boldsymbol{\Sigma}_{\mathbf{k}}^{\sigma}(\omega)\right]^{-1} .
$$

In analogy to the normal state the lowest-order approximation for the self-energy can be written as [29, 36]

$$
\boldsymbol{\Sigma}_{\mathbf{k}}^{\sigma}\left(i \omega_{n}\right)=\frac{-3}{N \beta} \sum_{\mathbf{q}, m} m_{\mathbf{k q}}^{2} \mathbf{G}_{\mathbf{k}-\mathbf{q}}^{\sigma}\left(i \omega_{m}\right) \chi_{\mathbf{q}}\left(i \omega_{n}-i \omega_{m}\right)
$$

where $i \omega_{n}=i \pi(2 n+1) / \beta$. Again neglecting the $\mathbf{k}$ dependence within each band, i.e., $\boldsymbol{\Sigma}_{\mathbf{k}}^{\sigma}=\boldsymbol{\Sigma}^{\sigma}$ one gets from Eq. (3) a nonzero gap $\Delta^{\sigma} \sim Z^{\sigma} \Sigma_{12}^{\sigma}(0)$, and

$$
\Delta^{\sigma}=\frac{-3 \lambda}{N} \sum_{\mathbf{q}} \chi_{\mathbf{q}}^{0} C_{\mathbf{q}}^{\bar{\sigma}} \frac{Z^{e} Z^{h} \Delta^{\bar{\sigma}}}{2 E_{\mathbf{q}}^{\bar{\sigma}}} \operatorname{th} \frac{\beta E_{\mathbf{q}}^{\bar{\sigma}}}{2}
$$

where $\left(E_{\mathbf{q}}^{e}\right)^{2}=\varepsilon_{\mathbf{q}}^{2}+\left(\Delta^{e}\right)^{2},\left(E_{\mathbf{q}}^{h}\right)^{2}=\varepsilon_{\mathbf{q}-\mathbf{Q}}^{2}+\left(\Delta^{h}\right)^{2}$ and $C_{\mathbf{q}}^{\bar{\sigma}}=I_{\mathbf{q}}^{\bar{\sigma}}\left(i \omega_{n} \sim 0\right) / \tilde{I}_{\mathbf{q}}^{\bar{\sigma}}$ plays the role of the cutoff function with

$$
I_{\mathbf{q}}^{\sigma}\left(i \omega_{n}\right)=\frac{1}{\beta \chi^{0}} \sum_{m} \chi\left(i \omega_{n}-i \omega_{m}\right) \frac{1}{\omega_{m}^{2}+\left(E_{\mathbf{q}}^{\sigma}\right)^{2}},
$$

and $\tilde{I}_{\mathbf{q}}^{\sigma}=\operatorname{th}\left(\beta E_{\mathbf{q}}^{\sigma} / 2\right) /\left(2 E_{\mathbf{q}}\right)$.

Finally, at $T=0$ Eqs. (25), (26) reduce to

$$
\Delta^{\sigma}=-\frac{3}{2} \lambda \chi^{0} Z^{\sigma} \Delta^{\bar{\sigma}} \int_{\omega_{c}^{\bar{\sigma}}}^{\omega_{c}^{\bar{\sigma}}} d \varepsilon \frac{\mathcal{N}^{\bar{\sigma}}(\varepsilon)}{\sqrt{\varepsilon^{2}+\left(\Delta^{\bar{\sigma}}\right)^{2}}}
$$

where $\omega_{c}^{\sigma}$ are effective cutoffs. It is evident from Eq. (27) that $\mathrm{SC}$ is of the $s_{ \pm}$- type [8], that is $\Delta^{h}=-\eta \Delta^{e}, \eta>0$. Assuming also $\mathcal{N}^{\sigma}(\varepsilon) \sim \mathcal{N}^{\sigma}$ we get from Eqs. 27), (8),

$$
1=\tilde{\gamma}^{e} \tilde{\gamma}^{h} B^{2} \ln \frac{\omega_{c}^{h}}{\Delta^{h}} \ln \frac{\omega_{c}^{e}}{\Delta^{e}},
$$

with $B=(4 / \pi) \ln \left(\omega_{0} / 2 T\right)$ connecting qualitatively the $\mathrm{N}$ state transport parameters with the gap equation. Clearly the SC Eliashberg equations are treated in a simplified manner [37] in order to get the familiar BCS-type form. Still the message is quite clear: spin-fluctuation mediated interaction gives naturally the $s_{ \pm}$- type SC pairing consistent with other approaches [8]. Parameters entering Eq. (28) are besides $B \sim \mathcal{O}(1)$ (depending on the form of the spin-fluctuation spectra) the cutoffs $\omega_{c}^{\sigma}$ determined by the characteristic spin fluctuation frequency and $\tilde{\gamma}^{\sigma}$. The latter have clearly the strongest influence and according to our estimates from transport, Eq. 22), $\tilde{\gamma}^{\sigma}>1$ are large requiring the strong coupling approach both for the $\mathrm{N}$ and for the SC state. As discussed in the next section on the basis of emerging single-crystal results, smaller resistivities $\rho(T)$ are reported and consequently $\tilde{\gamma}<1$. Still $\tilde{A}_{0}$ and the coupling remain at least moderate and in the same range as in optimum-doped cuprates, giving support for, or at least not contradicting, the notion for an SC pairing mechanism and its strength common to both IP and cuprates. 


\section{DISCUSSION}

We have presented a theory based on the spin-fluctuation induced coupling between the e- and h-bands in IP with the motivation to explain their anomalous $\mathrm{N}$-state transport properties. Existing experimental data on polycrystalline samples indicate that the QP damping and transport relaxation rates are even higher than in underdoped cuprates. It seems rather unlikely that quantitatively similar results should obtain for single crystals, as evidenced quite recently by measurements on, e.g., $\mathrm{BaFe}_{2}\left(\mathrm{As}_{1-\mathrm{x}} \mathrm{P}_{\mathrm{x}}\right)_{2}$, a material from the 122 family of IP [38] or $\mathrm{BaFe}_{2-\mathrm{x}} \mathrm{Co}_{\mathrm{x}} \mathrm{As}_{2}$ [19] where $\tilde{\gamma}$ and $1 / \mu$ are substantially reduced with respect to values in (polycrystalline) LFAO samples of comparable doping. However, the marked linearity of $\rho(T) \sim T / A_{0}+$ const. observed in $\mathrm{BaFe}_{2}\left(\mathrm{As}_{1-\mathrm{x}} \mathrm{P}_{\mathrm{x}}\right)_{2}$ over most of the doping region with nonzero $T_{c}$ testifies to the non-FL behavior, similar to cuprates. Likewise the evolution with doping in LFAO compounds is quite analogous, from a near insulator in an undoped substance to a FL-like behavior in the overdoped IP. Observe, however, that for the $\mathrm{NdFe} \mathrm{AsO}_{1-\mathrm{x}} \mathrm{F}_{\mathrm{x}}$ compound there is not much difference between polycrystalline and single crystal data concerning $\rho(T)$ and, e.g., $\alpha$, Eq. (22). Thus, for $x=0.18$ single crystal sample [39] a rough estimate for $T \sim 200 \mathrm{~K}-300 \mathrm{~K}$ yields $\alpha \sim 0.023$ whereas in a polycrystalline sample $x=0.11$ [40] $\alpha \sim 0.009$, resulting in $\tilde{\gamma} \sim 0.5$ and 1.1, respectively, i.e., again comparable to cuprates. Thus a more systematic study of the transport and optical properties in single crystal compounds of the separate families of oxypnictides is needed to settle this issue, particularly in view of the recent analysis of the competition between the linear and quadratic in $T$ contributions to $\rho(T)$, where the former is seen to dominate $\rho(T)$ in samples with the highest $T_{c}$ for the compounds there considered [2, 41].

Certain conclusions emerging from the above analysis still seem to be hard to avoid: a) the coupling to spin fluctuations is apparently substantial so that the QP damping is large with the QP overdamped in the low-doping regime, b) the effect of spin fluctuations on the N-state transport and on the SC pairing likewise appears to be strong implying pronounced spin fluctuations in the low-frequency window, both properties shared by underdoped and optimally doped cuprates as well, c) if estimates emerging from experiments are correct the strength of the coupling could be beyond the applicability of the lowest-order perturbation theory employed here, $d$ ) the behavior of IP even at modest $T>T_{c}$ is non-FL-like as clearly evidenced by large $S(T)$ as well as the $T$-dependence of $R_{H}(T)$, whereby the common features with cuprates stem from the large spin-fermion coupling and not from the MottHubbard physics. However, rather scarce experimental evidence for low-energy spin fluctuations requires some caution and additional efforts to pin down the proper ingredients for a viable theory of IP.

\section{ACKNOWLEDGEMENTS}

Authors acknowledge fruitful discussions with C. Hess and the access to their unpublished data, and the financial support of MHEST and JPSJ under the Slovenia-Japan Research Cooperative Program. T.T. acknowledges the support of the TRIP project.

[1] Y. Kamihara, T. Watanabe, M. Hirano, and H. Hosono, J. Am. Chem. Soc. 130, 3296 (2008).

[2] for an experimental overview see K. Ishida, Y. Nakai, and H. Hosono, J. Phys. Soc. Jap. 78, 062001 (2009).

[3] M. Imada, A. Fujimori, and Y. Tokura, Rev. Mod. Phys. 70, 1039 (1998).

[4] A. S. Sefat et al., Phys. Rev. B77, 174503 (2008).

[5] S. C. Lee et al., J. Phys. Soc. Jpn. 78, 043703 (2009).

[6] C. Hess et al., arXiv:0811.1601v1.

[7] K. Hashimoto et al., Phys. Rev. Lett. 102, 017002 (2009).

[8] for a theoretical overview see I. I. Mazin and J. Schmalian, arXiv:0901.4790

[9] H. Mukuda et al., arXiv:0904.4301 1.

[10] C. de la Cruz et al., Nature 453, 899 (2008).

[11] Y. Ando et al., Phys. Rev. Lett.87, 017001 (2001); Phys. Rev. Lett.93, 267001 (2004).

[12] H. Eisaki et al., J. Phys. Soc. Jpn. 77, Suppl. C, 36 (2008); K. Miyazawa et al., J. Phys. Soc. Jpn. 78, 034712 (2009).

[13] J. R. Cooper and J. W. Loram, J. Phys. I (France), 6, 2237 (1996).

[14] Y. Kohama et al., Europhys. Lett.84, 37005 (2008).

[15] M. M. Qazilbash et al., arXiv:0808.3748v1.

[16] C. M. Varma et al., Phys. Rev. Lett.63, 1996 (1989).

[17] K. Haule and H. Kotliar, New J. Phys. 11, 025021 (2009).

[18] Y. Nakajima, T. Taen, and T. Tamegai, J. Phys. Soc. Jap. 78, 023702 (2009).

[19] L. Fang et al., arXiv:0903.2418 2; F. Rullier-Albenque, D. Colson, A. Forget, and H. Alloul, arXiv:0903.5243v1.

[20] S. Raghu, X.-L. Qi, C.-X. Liu, D. J. Scalapino and S.-C. Zhang, Phys. Rev. B77, 220503(R) (2008).

[21] W.-Q. Chen, K.-Y. Yang, Y. Zhou, and F.-C. Zhang, Phys. Rev. Lett. 102, 047006 (2009).

[22] M. M. Korshunov and I. Eremin, Europhys. Lett. 83, 67003 (2008).

[23] S. Graser, T. A. Mayer, P. J. Hirschfeld, and D. J. Scalapino, New J. Phys. 11, 025016 (2009).

[24] A. V. Chubukov and D. K. Morr, Phys. Rep. 288, 355 (1997).

[25] D. J. Singh and M.-H. Du, Phys. Rev. Lett. 100, 237003 (2008).

[26] C. Liu et al., arXiv:0806.2147v2.

[27] A. I. Coldea et al., Phys. Rev. Lett. 101, 216402 (2008).

[28] J. Jaklič and P. Prelovšek, Adv. Phys. 49, 1 (2000).

[29] P. Prelovšek and A. Ramšak, Phys. Rev. B65, 174529 (2002).

[30] M. M. Zemljič and P. Prelovšek, Phys. Rev. B72, 075108 (2005).

[31] P. Prelovšek, I. Sega, and J. Bonča, Phys. Rev. Lett.92, 027002 (2004); I. Sega and P. Prelovšek, Phys. Rev. B 79, 140504(R) (2009).

[32] M. A. Kastner, R. J. Birgeneau, G. Shirane, and Y. Endoh, Rev. Mod. Phys. 70, 897 (1988).

[33] Y. Sekiba et al., New J. Phys. 11, 025020 (2009).

[34] G. D. Mahan, Many-Particle Physics, Kluwer Academic 
(2000).

[35] G. Palsson and and G. Kotliar, Phys. Rev. Lett.80, 4775 (1998).

[36] N. M. Plakida and V. S. Oudovenko, Phys. Rev. B59, 11949 (1999).

[37] O. V. Dolgov, I. I. Mazin, D. Parker, and A. A. Golubov, Phys. Rev. B 79, 060502(R) (2009).

[38] S. Kasahara et al., arXiv:0905.4427 1
[39] P. Cheng, H. Yang, Y. Jia, X. Zhu, G. Mu, and H.-H. Wen, Phys. Rev. B78, 134508 (2008).

[40] Z.-A. Ren et al., Europhys. Lett. 82, 57002 (2008).

[41] N. Doiron-Leyraud, P. Auban-Senzier, S. Rene de Cotret, A. Sedeki, C. Bourbonnais, D. Jerome, K. Bechgaard, and L. Taillefer, arXiv:0905.0964 1 . 